\title{
Myosin heavy-chain mutations that disrupt Caenorhabditis elegans thick filament assembly
}

\author{
Amy Bejsovec and Philip Anderson ${ }^{1}$ \\ Department of Genetics, University of Wisconsin, Madison, Wisconsin 53706 USA
}

\begin{abstract}
We have investigated Caenorhabditis elegans mutants in which altered unc-54 myosin heavy-chain protein interferes with assembly of thick myofilaments. These mutants have a dominant, muscle-defective phenotype, because altered myosin heavy-chain B (MHC B), the product of the unc-54 gene, disrupts assembly of wild-type MHC B. The mutant MHC B also interferes with assembly of wild-type myosin heavy-chain A (MHC A), the product of another MHC gene expressed in body-wall muscle cells. Because of disrupted MHC A assembly, dominant unc-54 mutants also exhibit a recessive-lethal phenotype. Dominant unc-54 mutations are missense alleles, and the defects in thick filament assembly result from mutant protein that is of normal molecular weight. Accumulation of mutant MHC B in amounts as little as $2 \%$ of wild-type levels is sufficient to disrupt assembly of both wild-type MHC A and MHC B. Dominant unc-54 mutations occur at remarkably high frequency following ethylmethane sulfonate (EMS) mutagenesis; their frequency is approximately equal to that of recessive, loss-of-function mutations. This unusually high gain-of-function frequency implies that many different amino acid substitutions in the myosin heavy-chain B protein can disrupt thick filament assembly.
\end{abstract}

[Key Words: Myosin; Caenorhabditis elegans; thick filament assembly; dominant mutations; unc-54]

Received April 15, 1988; revised version accepted August 18, 1988.

Assembly of the myofibrillar lattice requires that structural components of muscle interact in a precise fashion. Biochemical studies have defined these components (for review, see Squire 1981) and immunomicroscopy has assigned their locations within the sarcomere (for example, see Sanger et al. 1984; Francis and Waterston 1985; Maruyama et al. 1985; Bennett et al. 1986). The mechanisms by which the myofibrillar lattice is assembled, however, are largely unknown. Genetic techniques have been instrumental to our understanding of other problems of macromolecular assembly, such as bacteriophage morphogenesis (Jarvik and Botstein 1973; Wood and King 1979) and assembly of flagella in Chlamydomonas (Luck et al. 1982). Similar approaches should be valuable for the study of muscle assembly and function. Genetic analyses of muscle development have been undertaken primarily in Caenorhabditis elegans (Waterston et al. 1980; Zengel and Epstein 1980) and Drosophila melanogaster (Mogami and Hotta 1981; Deak et al. 1982; Karlik et al. 1984; Mogami et al. 1986). Both organisms have muscle tissues that can be mutated without affecting the viability of the animal.

Genetic analysis of muscle presents a special problem, because many muscle proteins are encoded by multigene families (for example, see Fyrberg et al. 1980; Bautch and

\footnotetext{
${ }^{1}$ Corresponding author.
}

Storti 1983; Minty et al. 1983; Romans and Firtel 1985). A mutation that reduces or eliminates gene function may have no phenotypic effect because other genes in the family supply a redundant function. In this case, dominant or incompletely dominant mutations are especially valuable (Suzuki 1970; Greenwald and Horvitz 1980). Dominant mutations may result in a visible phenotype if they create a novel function, inappropriately express the wild-type protein, or produce a disruptive product that interferes with the wild-type function (neomorphic, hypermorphic, and antimorphic mutations, respectively, in the nomenclature of Muller 1932). Dominant mutations have aided in characterizing multigene families of filamentous proteins in both $C$. elegans (Waterston et al. 1984) and Drosophila (Kemphues et al. 1979; Karlik et al. 1984; Hiromi and Hotta 1985).

The nematode C. elegans has four different muscle myosin heavy-chain (MHC) genes, and these encode four different MHC proteins (Epstein et al. 1974; Waterston et al. 1982; Miller et al. 1986). Heavy chains C and D are specific to the pharynx, while heavy chains $A$ and $B$ are expressed primarily in body-wall muscle cells (Mackenzie et al. 1978; Epstein et al. 1982). Heavy chains A and B assemble as homodimers (Schachat et al. 1978). Figure 1 summarizes the location of MHCs A and B within body-wall thick filaments. MHC A is restricted to the central region of thick filaments, while MHC B 


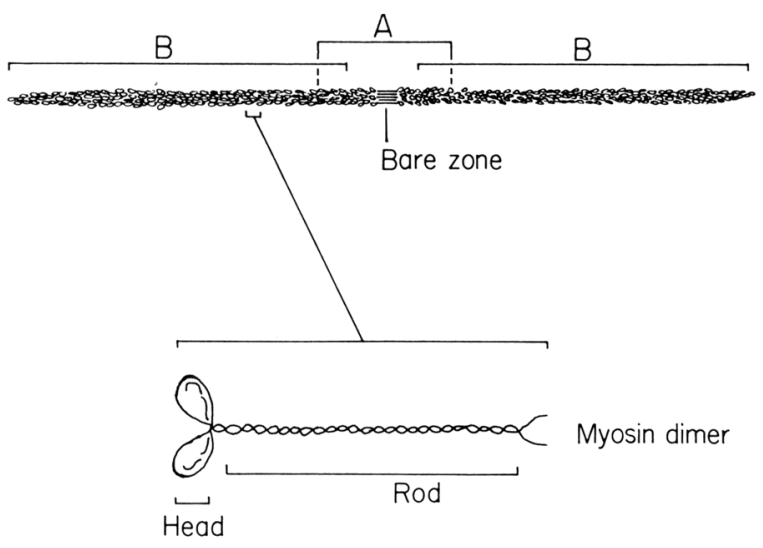

Figure 1. Schematic representation of C. elegans body-wall thick filaments. Zones $A$ and $B$ refer to regions of individual thick filaments that contain either MHC A, MHC B, or both (Miller et al. 1983). The thickness of the filament and the size of the bare zone are exaggerated in proportion to the length of the filament, which is about $10 \mu$. Each globular head contains one molecule of regulatory and alkali myosin light chain proteins.

overlaps partially with MHC A and extends to the filament termini (Miller et al. 1983). The MHC A-containing region includes the 'bare zone,' a region where myosin molecules interact in antiparallel fashion. Myosin interactions in this region are important for nucleating thick filament assembly (Niederman and Peters 1982).

unc-54 is the structural gene for MHC B, and several different classes of unc-54 mutations have been previously described. Mutants of the first class, unc-54 null alleles, are very frequent following ethylmethane sulfonate (EMS) mutagenesis. They accumulate no detectable MHC B and exhibit a muscle-defective, paralyzed (uncoordinated) phenotype (Epstein et al. 1974). Such mutations are recessive; loss-of-function heterozygotes are behaviorally wild type, although subtle ultrastructural irregularities can be distinguished in muscle cells. Mutations of a second class are also recessive, but occur at much lower frequency. They accumulate nearly normal amounts of a full-length MHC B and assemble a well-ordered myofilament lattice. The animals are slow, however, because of abnormal myosin function (Moerman et al. 1982; Dibb et al. 1985). Mutations of a third class contain small, weakly dominant deletions. These mutants accumulate nearly normal amounts of a MHC B that is shortened due to internal deletion (MacLeod et al. 1977; Dibb et al. 1985). Mutations of a fourth class are strongly dominant (MacLeod et al. 1977; Waterston et al. 1984; Dibb et al. 1985). Although certain atypical alleles of this class accumulate normal amounts of full-length MHC B (MacLeod et al. 1977; Dibb et al. 1985), the most frequent type of dominant mutations accumulate very little MHC B (see below).

We report here further properties of unc-54-dominant mutations. The mutant MHC B of these alleles fails to assemble into thick filaments, and its presence in muscle cells inhibits the assembly of wild-type MHC B. The genetic properties of these mutations and the ultrastructure of their affected muscle demonstrate that the mutant MHC B interferes not only with the assembly of wild-type MHC B but also with that of wild-type MHC A. As a result, these unc-54 mutations exhibit a recessive-lethal phenotype. Two features of these unc-54 mutations are particularly surprising: (i) accumulation of mutant MHC B in amounts as little as $2 \%$ of wild-type levels is sufficient to disrupt thick filament assembly; and (ii) following EMS mutagenesis, such dominant mutations arise at a frequency approximately equal to that of recessive, loss-of-function unc-54 mutations.

\section{Results}

\section{Dominant unc-54 mutations}

We have isolated over 40 unc-54-dominant mutations. We designate these alleles unc-54(d) in order to distinguish them from recessive, loss-of-function mutations, designated unc-54(0). The mutations were assigned to unc-54 on the basis of two criteria: (1) they map to the unc-54 region of linkage group $I$, and (2) in cis-trans tests they fail to complement unc-54(0) mutations. Since unc-54(d) mutations are incompletely dominant (see below), we are able to perform complementation tests with unc-54 recessive alleles. unc-54(d)/unc-54(0) trans-heterozygotes have a muscle defective phenotype much more severe than unc-54(d)/ + unc-54(do) double mutants are fully recessive (see below).

Table 1 summarizes the behavioral phenotypes of unc-54(d) mutants. Whereas unc-54 null alleles are fully recessive (Table 1, lines 2 and 3), unc-54(d)/ + heterozygotes are muscle defective and paralyzed (Table 1, line 4). The degree of paralysis varies among the different alleles, but generally unc-54(d)/ + heterozygotes are less severely paralyzed than unc-54(0) homozygotes. unc-54(d) mutations are properly described as incompletely dominant or semi-dominant, although we will refer to them simply as dominant.

Most unc-54(d) mutations are lethal when homozygous (Table 1, line 5; Dibb et al. 1985), dying as late embryos or very young larvae. Lethality appears to result from an absolute lack of body-wall muscle function. Mutant embryos generally arrest at the three-fold stage, emerge from the egg, but fail to unfold from their embryonic position. Pharyngeal pumping is normal in arrested embryos.

The lethality of many unc-54(d) homozygotes is suppressed by sup-3(e1407) (Table 1, line 7). sup-3 mutations are weak suppressors of all unc-54(0) alleles (Riddle and Brenner 1978). Suppression occurs because MHC A levels are increased approximately twofold in sup-3 mutants (Waterston et al. 1982). This overexpression of MHC A is due to rearrangements affecting the MHC A structural gene (Miller and Maruyama 1986). Of 24 unc-54(d) alleles tested, 15 are viable and paralyzed as unc-54(d); sup-3(e1407) double mutants.

The lethality caused by some unc-54(d) mutations is 
Table 1. unc-54(d) mutants are muscle defective when heterozygous and lethal when homozygous

\begin{tabular}{ll}
\hline Genotype & Motility phenotype \\
\hline$+/+$ & wild type \\
$\frac{\text { unc-54(0) }}{+}$ & wild type \\
$\frac{\text { unc-54(0) }}{\text { unc-54(0) }}$ & paralyzed \\
$\frac{\text { unc-54(d) }}{+}$ & $\begin{array}{c}\text { paralyzed; severity varies, depending on } \\
\text { allele }\end{array}$ \\
$\frac{\text { unc-54(d) }}{\text { unc-54(d) }}$ & $\begin{array}{c}\text { late embryonic or early larval lethal } \\
\text { unc-54(d) }\end{array}$ \\
eDf10 & $\begin{array}{c}\text { either severely paralyzed or lethal, } \\
\text { depending on allele }\end{array}$ \\
$\frac{\text { unc-54(d);sup-3 }}{\text { unc-54(d);sup-3 }}$ & $\begin{array}{c}\text { either severely paralyzed or lethal, } \\
\text { depending on allele }\end{array}$ \\
\hline
\end{tabular}

unc-54(0) and unc-54(d) represent recessive and dominant alleles of unc-54, respectively. The phenotypes of unc-54(0) mutants are typical for alleles that express no MHC B. The phenotypes of unc-54(d) mutants depend on the allele used, as indicated in the table.

$e D f 10$ is a deletion that eliminates the entire unc-54 gene (Anderson and Brenner 1984); it is recessive to wild type.

sup-3 mutations overexpress MHC A approximately twofold (Waterston et al. 1982).

dependent on the number of mutant gene copies (Table 1, line 6). Certain mutations, for example unc-54(r371), are lethal when homozygous but viable when heterozygous to an unc-54 deficiency. Such animals, although viable, are severely paralyzed and have very low brood sizes. Other dominant mutations, for example unc-54(r342), are inviable when hemizygous. Of $12 \mathrm{mu}-$ tations tested, 8 are viable when hemizygous. Generally, the less strongly dominant alleles are those that are viable as hemizygotes.

Dominant unc-54 mutations are remarkably frequent. Following EMS mutagenesis, they occur at a frequency approximately equal to that of loss-of-function unc-54 mutations. In an experiment that was designed to recover both dominant and recessive unc- 54 mutations, we isolated approximately equal numbers of each type (see Table 2). We estimate the frequency of both dominant and recessive unc-54 mutations to be approximately $1.0 \times 10^{-3}$ per mutagenized gamete, a frequency slightly higher than that reported previously (Waterston et al. 1984). EMS-induced recessive mutations are very frequent, and they are distributed throughout the gene (Dibb et al. 1985). EMS-induced dominant mutations occur at an equally high frequency, indicating that their mutational target is also very large. These results indicate that many different nucleotide substitutions lead to an unc-54-dominant phenotype.

\section{unc-54(d) alleles are missense mutations}

Four lines of evidence, described below, indicate that unc-54(d) mutations result from amino acid substitutions in MHC B: (1) most unc-54(d) mutants express full-length $M H C B$, although at levels much less than in wild type; (2) unc-54(d)unc-54(0) double mutants are no longer dominant and no longer express MHC B; (3) unc-54(d) mutations exhibit no abnormalities on genomic Southern blots; and (4) typical unc-54(d) mutations accumulate near-normal amounts of full-length unc-54 mRNA.

Figures 2 and 3 show the myosin heavy-chain proteins accumulated by representative unc-54(d) alleles. The amount of MHC B accumulated by different alleles is variable, ranging from below the limits of detection to $\sim 30 \%$. The amount of MHC B accumulated by any single allele is reproducible and characteristic for that allele. For example, in Figure 2, lanes 3, 5, and 7 show unc-54(d) alleles that accumulate approximately 2, 5, and $20 \%$ of the wild-type level of MHC B, respectively. The low level of mutant MHC B in 1342 (lane 3) is rarely visible when total worm homogenate is electrophoresed. However, levels of $2 \%$ can be detected when the myosin heavy chains are immunoprecipitated with polyclonal anti-actomyosin antibodies (Fig. 3). It is important to note that MHC B levels can be quantitated only for those mutants that are viable as unc-54(d);sup-3 homozygotes. The mutant MHC B protein cannot be separated from wild-type protein for those alleles that must be maintained as heterozygotes. unc-54(d)/ + heterozygotes accumulate an amount of MHC B that is approximately half that of wild type, similar to unc-54(0)/ + heterozygotes. Thus, the stability of wild-type MHC B is not affected by the presence of mutant MHC B in dominant heterozygotes.

We examined unc-54(d) mutants for accumulation of MHC B-related polypeptides that might be shorter than full length. Actomyosin proteins were immunoprecipitated from unc-54(d);sup-3 mutants using a polyclonal serum that recognizes all MHC isoforms (R. Francis and R.H. Waterston, unpubl.). The immunoprecipitates were

Table 2. unc-54(d) mutations are remarkably frequent following EMS mutagenesis

\begin{tabular}{ccc}
\hline Genotype & Number isolated & $\begin{array}{l}\text { Mutation frequency } \\
\text { (mutations/gamete) }\end{array}$ \\
\hline$\frac{\text { unc-54(d) }}{+}$ & 6 & $1.0 \times 10^{-3}$ \\
$\frac{\text { unc-54(0) }}{+}$ & 8 & $1.3 \times 10^{-3}$
\end{tabular}

Wild-type (N2) animals were mutagenized with EMS. Among $3000 \mathrm{~F} 1$ offspring, we isolated six unc-54(d)/+ heterozygotes and eight unc-54(0)/ + heterozygotes. We identified unc-54(d)/+ heterozygotes by their muscle-defective, paralyzed phenotype and unc-54(0)/ + heterozygotes by scoring among the F2 offspring for the presence of unc-54(0) paralyzed homozygotes. 
Figure 2. Myosin heavy-chain proteins accumulated by unc-54(d) mutants. The $3.75 \%$ SDS-PAGE of total nematode protein resolves the myosin heavy chains. Only the high molecular weight region of the gel is shown. MHC A is present in all strains, and is elevated approximately twofold in strains that carry a sup-3(e1407) mutation. MHC B is absent in the recessive, null mutant unc-54(e190) [cf. lanes 1 (wild type) and 2]. unc-54(r371) and unc-54(r383) accumulate small amounts of full-length MHC B (e.g., lanes 5 and 7). The MHC B in these strains is indicated with arrows. unc-54(r342) accumulates a very low level of MHC B, but it was not resolved from MHC A in this experiment. The mutant MHC B proteins are absent in nondominant derivatives of these alleles (cf. lane 5 with 6 , and lane 7 with 81 .

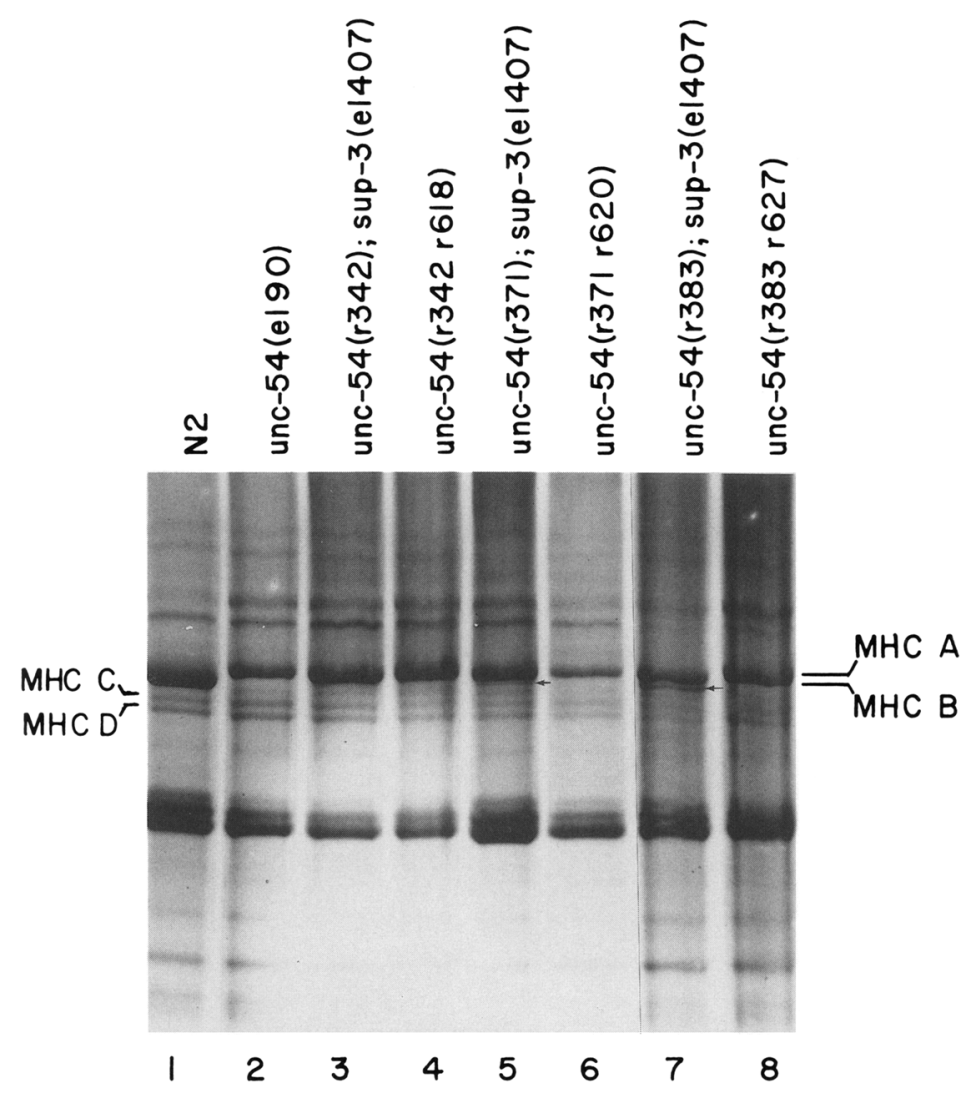

(MacLeod et al. 1977). Compared to the alleles discussed here, however, they are among the least dominant unc-54(d) mutations. The protein levels of other alleles that are inviable when homozygous (Waterston et al. 1984) had not been previously determined (R.H. Waterston, pers. comm.).

If the dominance of unc-54(d) mutations is mediated by a mutant MHC B polypeptide, then unc-54 loss-offunction mutations located in cis should eliminate the disruptive product; such double mutants should no longer be dominant. We have shown that this is true for three unc-54(d) alleles. We mutagenized balanced heterozygotes [genotypes unc-54(d) $+/+$ Let $(e 2000)]$ and isolated wild-type Fl offspring. In each case, these nondominant derivatives proved to harbor recessive, loss-of-

Figure 3. unc-54(d) mutants show allele-specific differences in the amount of accumulated MHC B protein. Proteins were immunoprecipitated with polyclonal anti-actomyosin antibodies (R. Francis and R.H. Waterston, unpubl.) and subjected to $3.75 \%$ SDS-PAGE. All mutants are unc-54(d);sup-3(e1407) homozygotes. The positions of MHCs A, B, C, and D are shown.

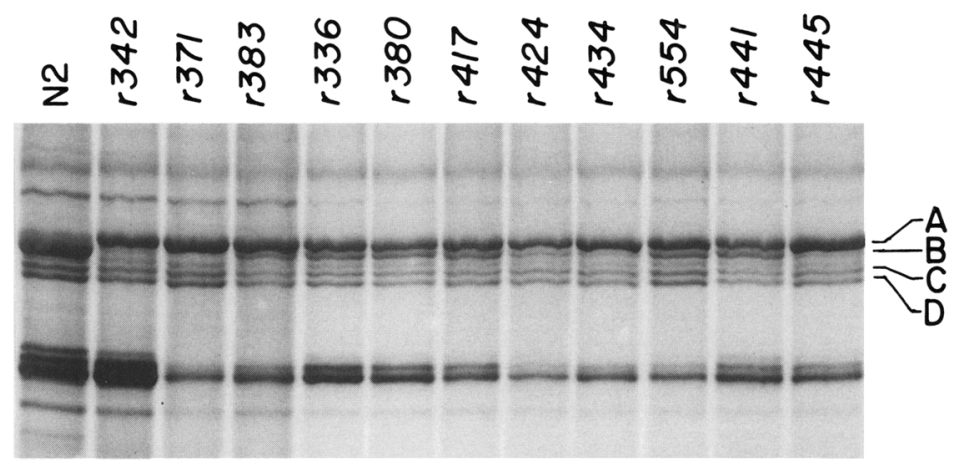


function unc-54 alleles. These unc-54 alleles are viable when homozygous and are identical to standard recessive unc-54 mutations in all respects. We infer that these unc-54 alleles are double mutants [genotype unc-54(d)unc-54(0)], in which an unc-54 loss-of-function mutation eliminates a disruptive gene product of the unc-54(d) mutation. Figure 2 demonstrates that the mutant MHC B proteins discussed above are absent in the nondominant derivatives (Fig. 2, cf. lanes 5 with 6 , and 7 with 8). We suspect that the dominance of unc-54(d) mutations, in all cases, is mediated by a mutant MHC B polypeptide, even for alleles where the amount of mutant MHC B is below our limits of detection.

unc-54(d) mutants have no abnormalities detectable on genomic Southern blots. We performed genomic Southern blots of 17 unc-54(d) mutations using a hybridization probe that covers the entire unc-54 gene. The pattern of restriction fragments was wild type for all mutants. unc-54(d) mutants express near-normal amounts of unc-54 mRNA. Using an unc-54 hybridization probe, we performed Northern blots for three unc-54(d) mutants. The size and abundance of unc-54 mRNA was normal for all mutants tested.

We conclude from the above results that unc-54(d) mutations are small lesions (affecting less than $50 \mathrm{bp}$ ), that most unc-54(d) mutants accumulate small amounts of full-length MHC B protein, and that the low levels of MHC B protein in unc-54(d) mutants are not due to low levels of unc-54 mRNA. The most likely EMS-induced mutations having these properties are single amino acid substitution (missense) alleles.

\section{unc-54(d) protein interferes with assembly of wild-type $M H C B$}

The ultrastructure of body-wall muscle in unc-54(d)/+ heterozygotes demonstrates that the mutant MHC B in'erferes with assembly into thick filaments of wild-type
MHC B. Before considering these data, we will review briefly the ultrastructure of wild-type body-wall muscle. A more thorough description of $C$. elegans muscle can be found in Waterston et al. (1980) and Francis and Waterston (1985).

Figure 4 shows a transmission electron micrograph of a transverse section of wild-type body-wall muscle. Because nematode muscle is obliquely striated (Rosenbluth 1965), each cross section reveals all elements of a sarcomere. Approximately two and one-half sarcomeres of a single body-wall muscle cell are shown in Figure 4. Each sarcomere is delimited by dense bodies, which extend from the overlying plasma membrane deep into the myofilament lattice. Surrounding each dense body is the I-band, containing only thin filaments. Thin filaments attach to the dense bodies and extend longitudinally into the sarcomere, where they interdigitate with thick filaments in the A-band. An $\mathrm{H}$-zone and $\mathrm{M}$-line are apparent in the center of each A-band. Thick filaments are approximately $10-\mu$ long and $25 \mathrm{~nm}$ in diameter, except near their termini where they taper to about $15 \mathrm{~nm}$.

Figure 5 compares the muscle tissue of representative unc-54(d)/ + and unc-54(0)/ + heterozygotes. unc-54(0)/ + heterozygotes are behaviorally wild type and Figure $5 \mathrm{~B}$ shows that their muscle ultrastructure closely resembles wild type. Thick filaments are fewer in number than wild type because of the reduced content of MHC B. unc-54(d)/ + heterozygotes (Fig. 5A) contain many fewer thick filaments than unc-54(0)/ + heterozygotes. Dense bodies are diffuse and irregularly spaced, and the myofilament lattice is generally disorganized. The number and orientation of thin filaments are normal. The sections pictured in Figure 5 were chosen because they are typical examples of unc-54(d)/ + and unc-54(0)/ + heterozygotes. We counted the number of thick filaments present in a constant cross-sectional area of 4 representative sections of each strain. unc-54(r371)/ + contained $305 \pm 31$ thick filaments (mean \pm standard

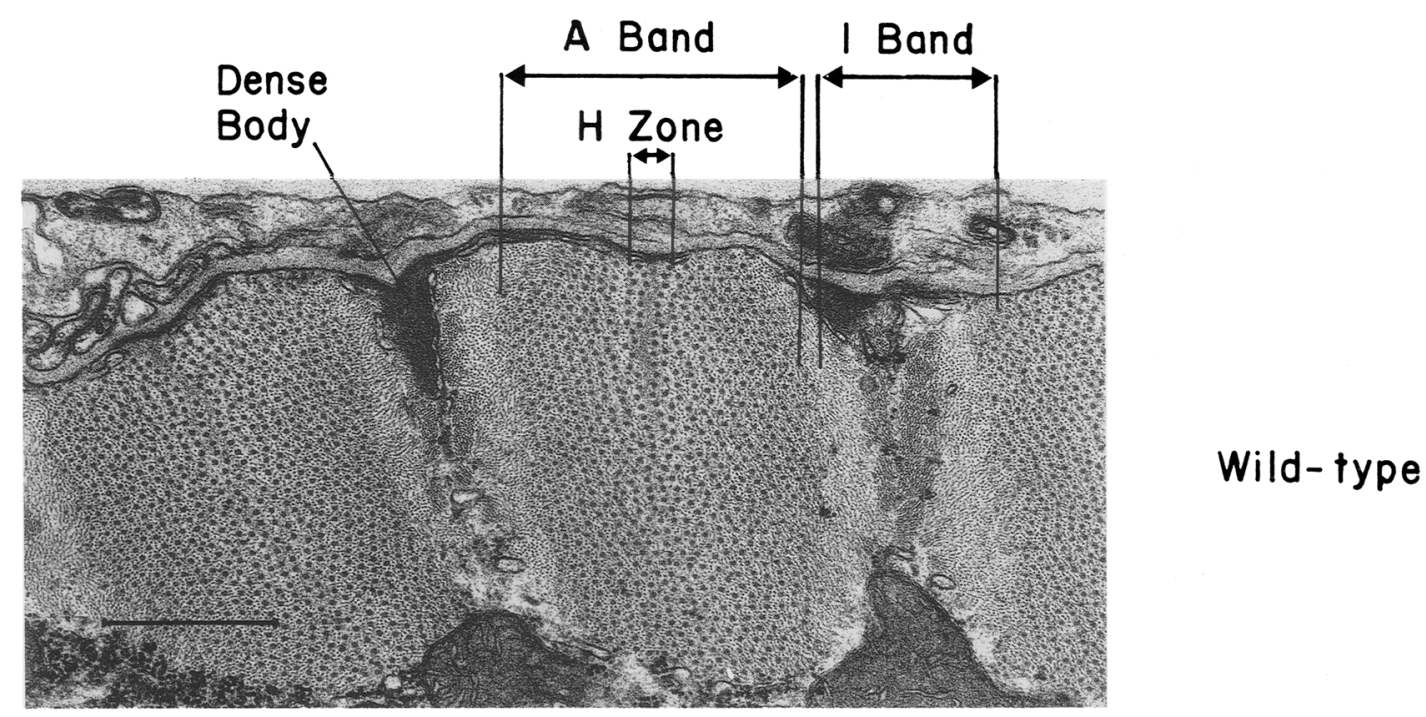

Figure 4. Transmission electron micrograph of a transverse section of wild-type body-wall muscle. The bar represents $0.5 \mu$ in this and all subsequent micrographs. 
A

B

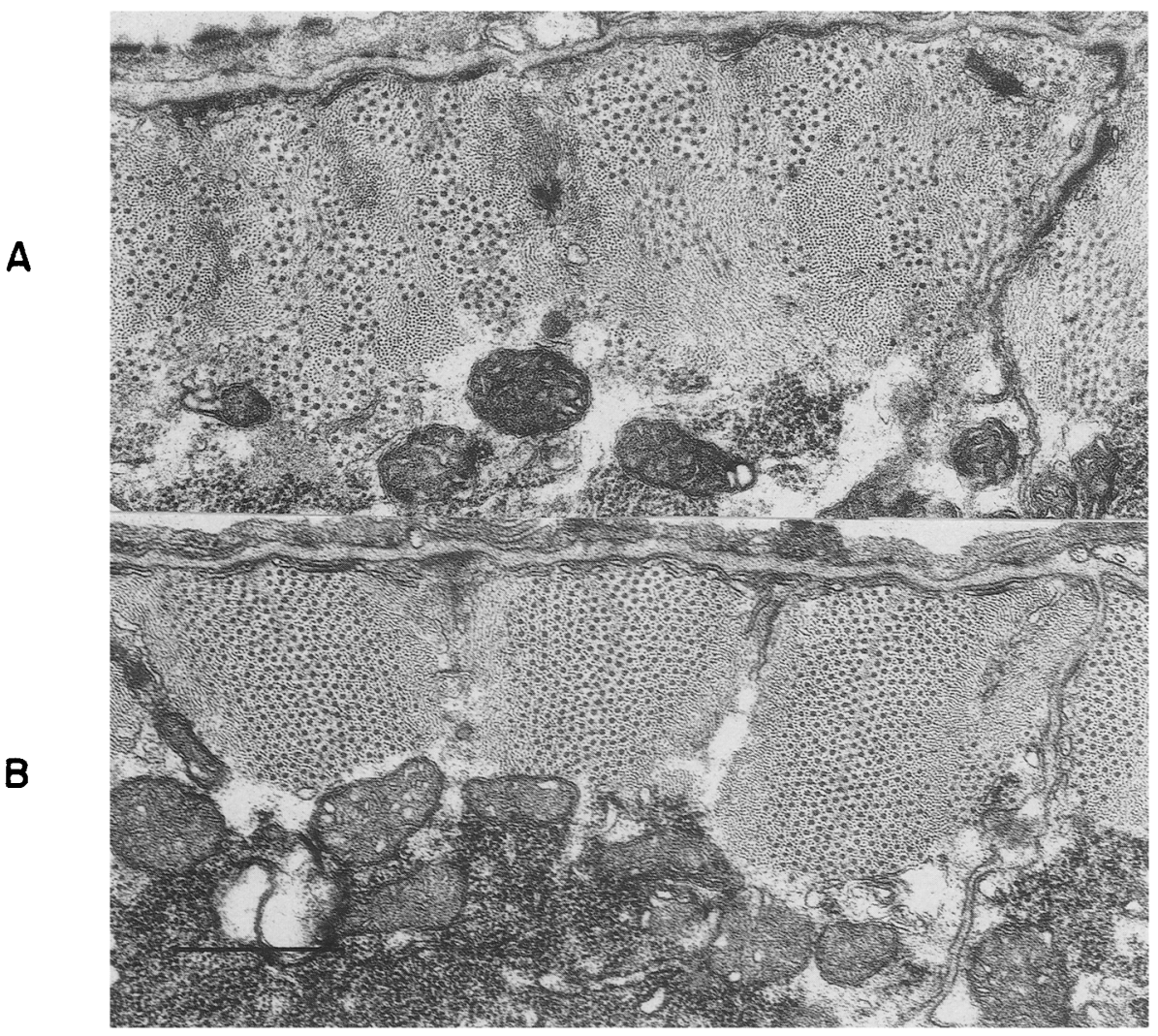

unc -54 (d) /t+

unc-54(0)/t

Figure 5. unc-54(d)/ + heterozygotes contain many fewer thick filaments than unc-54(0)/+ heterozygotes. $(A)$ A representative section of unc-54(r371)/ + (B) A representative section of $e D f 10 /+$. Both strains express equal amounts of wild-type MHC B. Mutant MHC B of the unc-54(d) gene, therefore, interferes with assembly into thick filaments of wild-type MHC B.

deviation); $e D f 10 /+$ (a recessive heterozygote) contained $833 \pm 83$ thick filaments. Since the animals pictured in Figure 5 contain the same levels of wild-type MHC B, we conclude that mutant MHC B of the unc-54(d) gene interferes with assembly of wild-type MHC B into thick filaments. The reduced number of thick filaments seen in cross sections of unc-54(d) heterozygotes could be because the filaments are either fewer in number or because they are shorter in length. We cannot distinguish between these possibilities.

unc-54(d) protein interferes with assembly of wild-type $M H C A$

Figure 6 compares the muscle tissues of representative unc-54(d);sup-3 and unc-54(0); sup-3 homozygotes. Since unc-54(0) mutations are null alleles, the thick filaments present in unc-54(0); sup-3 (Fig. 6B) are composed exclusively of MHC A. unc-54(d); sup-3 homozygotes contain many fewer thick filaments than unc-54(0); sup3 homozygotes. The sections pictured in Figure 6 were chosen because they are typical examples of these strains. We counted the number of thick filaments present in a constant cross-sectional area of 3 representative sections for each strain. unc-54(r371); sup- 3(e1407) contained $215 \pm 39$ thick filaments; unc-54(e190); sup-3(e1407) contained $320 \pm 12$ thick filaments. Since the level of MHC A is the same in these two strains, we conclude that the mutant MHC B interferes with assembly of wild-type MHC A. Again, this effect causes the filaments to be either fewer in number or shorter in length.

Figure 7 compares the muscle tissue of a representative unc-54(d)/eDf10 hemizygote with that of an unc-54(0) homozygote. Because unc-54(0) mutations are null alleles, the thick filaments present in unc-54(0) homozygotes (Fig. 7B) are composed exclusively of MHC A. The number of thick filaments in this strain reflects the wild type level of expression of MHC A. unc-54(d)/ $e D f 10$ hemizygotes (Fig. 7A) contain so few thick filaments that we were unable to accurately determine their number. As the level of MHC A is the same in these two strains, these results confirm our conclusion (above) that mutant MHC B of the unc-54(d) gene interferes with assembly of wild-type MHC A.

We have examined mutant muscle ultrastructure for three different unc-54(d) alleles (r342, r371, and r383). Heterozygotes, hemizygotes, and suppressed homozygotes all show a striking effect of the unc-54(d) mutation on thick filament number and organization. However, 


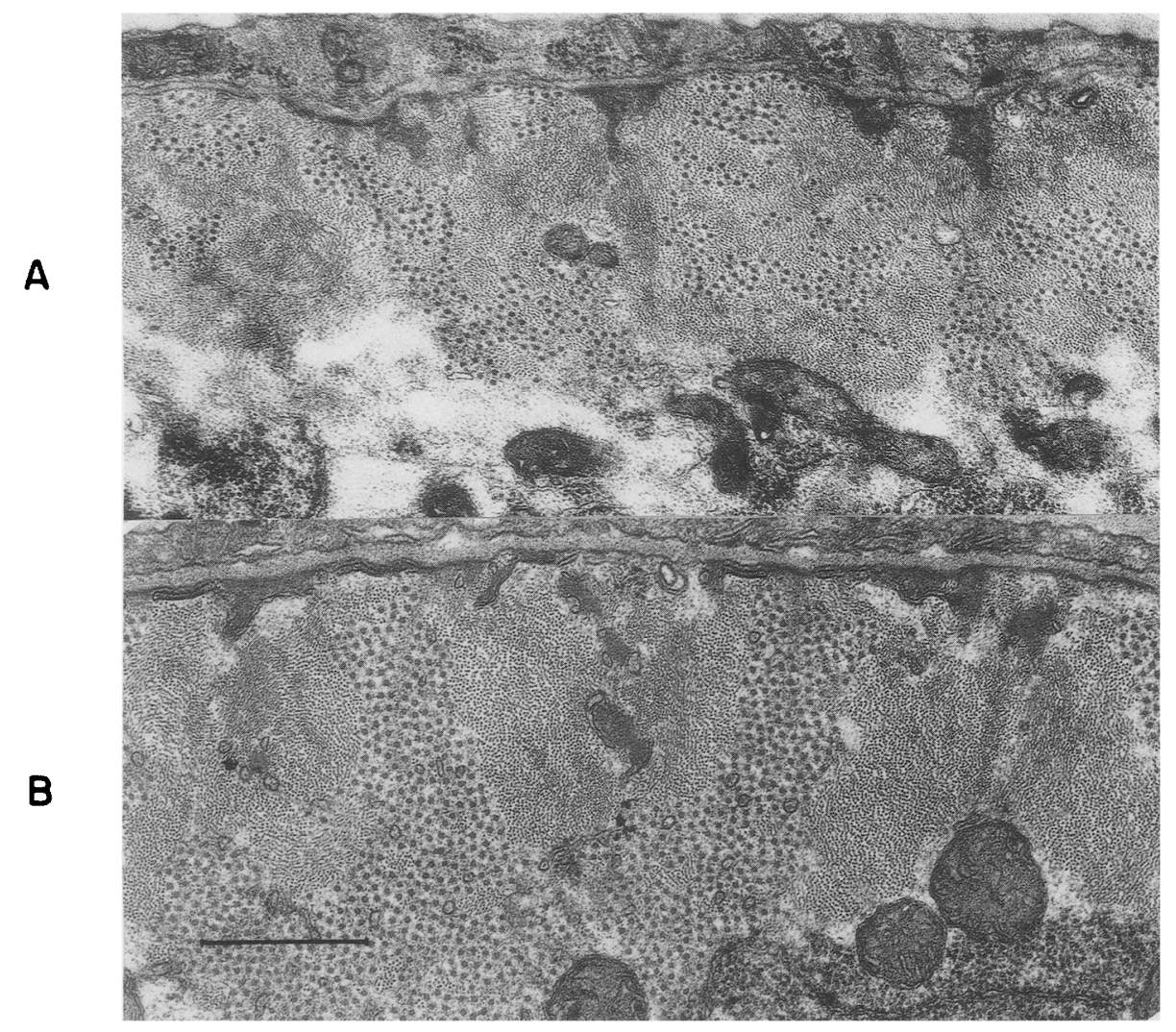

\section{unc-54(d); sup-3}

\section{unc-54(0); sup -3}

Figure 6. unc-54(d);sup-3 homozygotes contain many fewer thick filaments than unc-54(0); sup-3 homozygotes. Panel $A$ shows a representative section of unc-54(r371);sup-3(e1407). Panel $B$ shows a representative section of unc-54(e190);sup-3(e1407). Both strains express equal amounts of wild-type MHC A. Mutant MHC B of the unc-54(d) gene, therefore, interferes with assembly into thick filaments of wild-type MHC A.

the three alleles cause different degrees of ultrastructural disruption and this correlates directly with the severity of behavioral phenotype. $r 342$ mutants consistently have fewer thick filaments than $r 371$, which in turn have fewer thick filaments than $r 383$ mutants. $r 342$ is the most strongly dominant of these mutations and r383 the least strongly dominant.

\section{Discussion}

We describe here properties of unc-54 myosin heavychain mutations that disrupt the process of thick filament assembly. Myosin heavy-chain B, the product of the unc-54 gene, is abnormal in these mutants. The mutant MHC B fails to assemble into thick filaments, and its presence in muscle cells interferes with assembly of wild-type MHC A and B. Due to their effects on assembly of wild-type MHC B, these mutants have a dominant muscle-defective phenotype. Due to their effects on assembly of wild-type MHC A, these mutants have a recessive-lethal phenotype. unc-54(d) mutations are missense alleles, and their MHC B product is unstable in vivo. unc-54(d) protein, when present in quantities as low as $2 \%$ of wild-type amounts, is sufficient to cause these disruptive effects.
At what point during thick filament assembly might unc-54(d) mutations exert their effects? To produce a wild-type thick filament, myosin heavy chains must fold appropriately after their synthesis, they must coassemble with myosin light and heavy chains to form myosin dimers, and myosin dimers must assemble with each other and with additional proteins in the growing thick filament. In theory, unc-54 dominant mutations could disrupt any of these steps. We think it likely that the important disruptive effects of unc-54(d) mutations occur during assembly of the thick filament itself, after myosin dimer formation. unc-54(d) mutants accumulate very little mutant MHC B (as little as $2 \%$ of wild-type levels). Yet, this mutant MHC B has drastic effects on the assembly of a vast excess of wild-type myosins A and B. There is simply too little unc-54(d) protein for the disruption to occur at any other stage.

We envision two general mechanisms by which unc-54(d) protein inhibits assembly of both wild-type myosins A and B. The first involves mutant MHC B acting to disrupt initiation of thick filament assembly. Thick filament assembly initiates in the bare zone, where myosin dimers stack in an antiparallel fashion. Functions of the nonhelical tip of the MHC rod segment are important for antiparallel interactions (Kuznicki et 
A

B

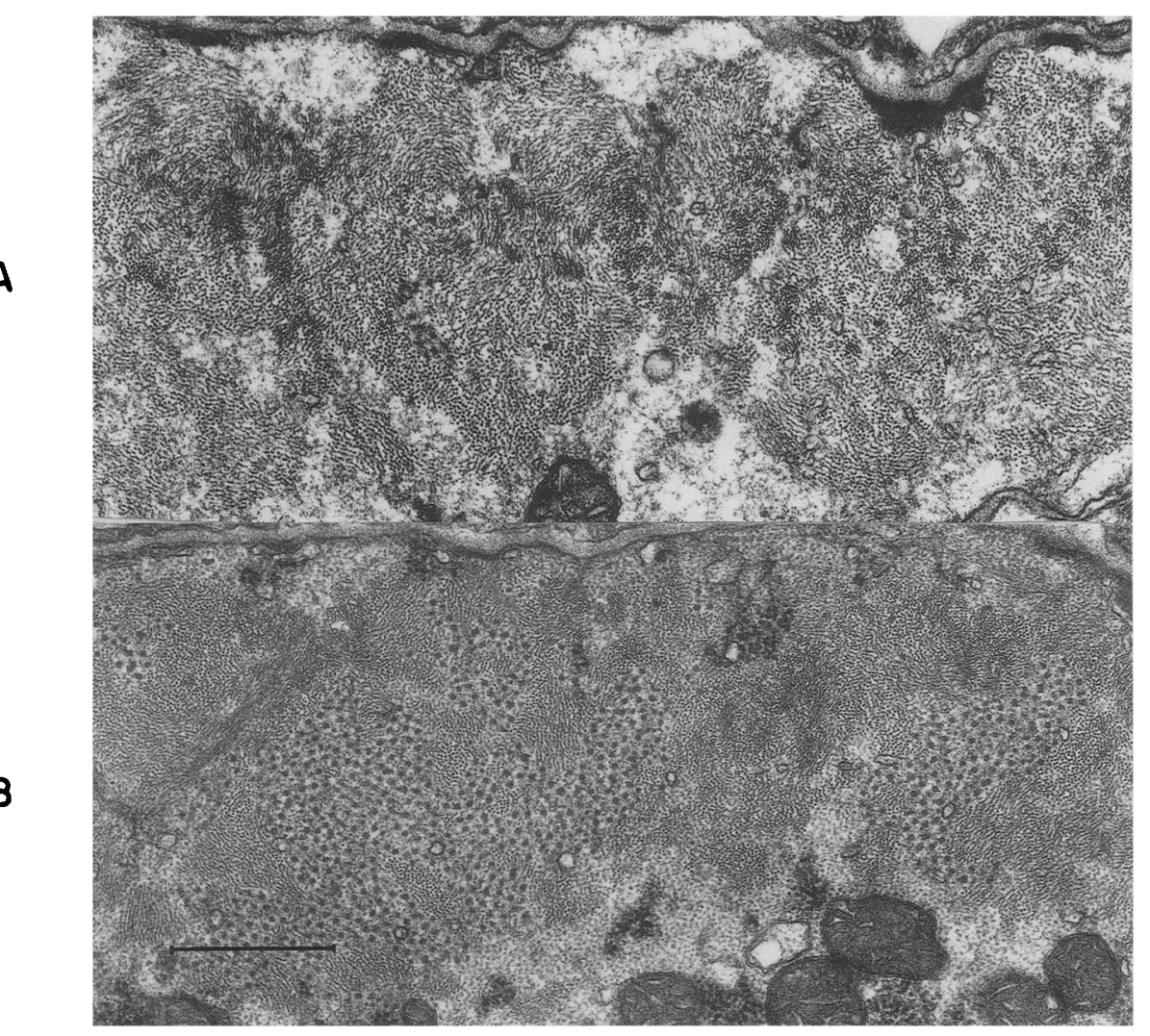

unc-54(d)/eDflo

unc-54(0)

Figure 7. unc-54(d)/eDf10 hemizygotes contain very few thick filaments. Panel $A$ shows a representative section of unc-54(r371)/ $e D f 10$. Panel $B$ shows a representative section of unc-54(e190). Both of these strains express equal amounts of wild-type MHC A. The mutant MHC B of the unc-54(d) allele, therefore, interferes with assembly into thick filaments of wild-type MHC A.

al. 1985; Pagh and Gerisch 1986). In C. elegans bodywall thick filaments, myosin A alone is localized to this region (Miller et al. 1983). Myosin A interactions at the bare zone, either with components of the M-line or with nonmyosin components of the thick filament (Epstein et al. 1985), may be important for initiating filament assembly. Perhaps the unc-54(d) mutant MHC B localizes inappropriately to this region (either as homodimers or as $\mathrm{A} / \mathrm{B}$ heterodimers), thereby disrupting the initial steps of thick filament assembly. According to this model, wild-type myosins $A$ and $B$ fail to assemble because there are fewer nucleated centers available for filament elongation.

The second general mechanism involves mutant MHC B acting to inhibit elongation of assembling thick filaments. Myosin, paramyosin, and components of the core structure (Epstein et al. 1985) are added to growing filaments during assembly. Perhaps unc-54(d) mutant myosin is able to incorporate into a growing filament, but once incorporated it prevents or interferes with the addition of further thick filament components. The result would be a truncated or 'capped' structure that is incapable of further elongation.

The two general models discussed above make testable predictions. If unc-54(d) mutations inhibit filament elongation but not initiation, then thick filaments in unc-54(d) mutants should be normal in number but shorter in length. If unc-54(d) mutations inhibit filament initiation but not elongation, then thick filaments should be fewer in number but normal in length. Experiments are in progress to distinguish between these possibilities.

Where within the myosin molecule are typical unc-54(d) mutations located? The high frequency of unc-54(d) mutations following EMS mutagenesis indicates that unc-54(d) mutations are distributed over a substantial portion of the MHC B molecule. The rod segment of myosin is known to be important for both parallel and antiparallel interactions (Kiehart et al. 1984; Kuznicki et al. 1985; Peltz et al. 1985; Pagh and Gerisch 1986). The alpha-helical rod segment, in common with many fibrous proteins, shows a repeating pattern of 7 amino acids (McLachlan and Karn 1982, 1983). The position of hydrophobic and charged amino acids within these repeats is crucial for establishing the coiled-coil structure and for aligning adjacent myosin dimers within the thick filament (McLachlan and Karn 1982, 1983). Perhaps amino acid substitutions that alter the repeat structure disrupt inter-chain or inter-dimer interactions, resulting in the assembly defects that we observe. Alternatively, unc-54(d) mutations might lie in the globular head region. Phosphorylation of the regulatory light-chain is important for assembly of smooth muscle and nonmuscle myosin filaments (Suzuki et al. 
1978; Kendrick-Jones et al. 1982). Craig et al. (1987) suggest that light chain phosphorylation activates the myosin molecule by altering interactions between adjacent heads or between heads and the filament backbone. Thus, functions of the head may be required for filament assembly and alterations of head structure might lead to assembly defects. Experiments are in progress to determine the locations and exact sequences of typical unc-54(d) mutations.

The DNA sequence of one partially dominant unc-54 mutation has been previously determined (Dibb et al. 1985). unc-54(e1152), causes two adjacent amino acid substitutions within the rod, very near its beginning. This mutation appears to disturb the rod either by affecting its charged surface or by altering its stability (Dibb et al. 1985). e1152 is atypical of unc-54(d) mutations, however, because it is viable when homozygous and accumulates a normal amount of mutant MHC B.

One of the most surprising features of unc-54(d) mutants is that such a small amount of mutant MHC B suffices to produce dramatic effects. unc-54(d) mutants that accumulate the least amount of MHC B $<2 \%$ wild-type levels) are the most strongly dominant; mutants that accumulate greater amounts of mutant MHC B generally show a less severe phenotype. Perhaps the amount of mutant MHC B that accumulates in unc-54(d) mutants reflects how much of it is incorporated into mutant thick filaments and, therefore, protected from degradation. Abnormal proteins are proteolyzed rapidly (for review, see Goldberg and St. John 1976; Hershko and Ciechanover 1982), but they may be stabilized when incorporated into a structure (Clark et al. 1986). The less strongly dominant alleles might express a less disruptive MHC B, where more molecules in total, or a greater number adjacent to each other, must be incorporated before assembly is aborted. The degradation of unassembled mutant MHC B, therefore, might be secondary to its primary defect concerning assembly.

In a broader context, the frequent occurrence of unc-54 dominant mutations may reflect a general property of proteins that are structural components of filaments or similar macromolecular complexes. For example, collagen, keratin, and troponin, among other proteins, exhibit a coiled-coil structure similar to that of the MHC rod segment. The assembly of complexes like muscle or the cytoskeleton may be very sensitive to mutational disruption. The spontaneous mutation rate of several autosomal dominant human disorders is in excess of $10^{-5}$ mutations per gamete per generation (Levitan 1988). Perhaps this high mutation rate results from the genetic properties of structural proteins like those described here.

\section{Materials and methods}

Nematode strains

Most strains used in this work are derived from the wild-type Bristol (N2) stock. The conditions for culturing C. elegans are described by Brenner (1974). The unc-54(d) mutations discussed here were induced with ethylmethane sulfonate (EMS). Many of our unc-54(d) alleles were isolated using a selection that enriches for muscle-defective uncoordinated mutants (Bejsovec et al. 1984; Park and Horvitz 1986). Others were identified directly by their heterozygous muscle-defective phenotype. unc-54(e190) is an EMS-induced deletion that expresses no MHC B (Epstein et al. 1974; MacLeod et al. 1981). eDf10 is an unc-54 deletion that eliminates the unc-54 gene and two nearby lethal complementation groups (Anderson and Brenner 1984).

\section{Frequency of unc-54(d) mutations}

To directly compare the frequencies of unc-54(d) and unc-54(0) mutations in a wild-type genetic background, a synchronized population of early L4 larvae was mutagenized with $50 \mathrm{~mm}$ EMS for $3.5 \mathrm{hr}$. Pools of $10 \mathrm{~F} 1$ progeny were established from these animals. The F1 progeny were screened, and animals exhibiting the characteristic unc-54(d)/+ phenotype were retained. Three days later, the F2 populations were screened for the presence of unc-54(0) homozygotes. To ensure that all mutations are independent, no more than one mutant was retained from each population of Fl animals. Complementation tests with canonical unc-54 alleles confirmed the genotypes of these mutants.

\section{Reversion of dominance}

The dominance of three unc-54(d) alleles was eliminated following EMS mutagenesis. unc-54(r342), unc-54(r371), and unc-54(r383) were balanced with Let $(e 2000)$. The Let $(e 2000)$ chromosome is wild-type for unc-54 and lethal when homozygous. Balanced heterozygotes have a muscle-defective, uncoordinated phenotype due to dominance of the unc-54(d) mutation. Heterozygotes were synchronized, and L4 larvae were mutagenized with EMS. Small pools of F1 progeny were established from single parents. These Fl pools were screened for motile, wild-type revertants. Wild-type Fl animals were isolated singly and their progeny scored for segregation of unc-54(0) homozygotes. Such animals have a characteristic paralyzed phenotype. Complementation tests with canonical unc-54 alleles verified the genotypes of these mutants. Several independent nondominant revertants were isolated for each of the unc-54(d) strains tested.

\section{Electron microscopy}

Thin sections were prepared for electron microscopy as described by Waterston et al. (1980) and Moerman et al. (1982). Animals chosen for sectioning were all young adults, the stage which occurs in the wild type at about $70 \mathrm{hr}$ after hatching. Sections were taken slightly posterior to the second pharyngeal bulb. A Phillips 410 electron microscope was used to view the specimens.

\section{Gel electrophoresis of proteins}

Proteins from whole nematode homogenates were solubilized in sodium dodecylsulfate (SDS) sample buffer. Myosin immunoprecipitates were obtained by sonicating nematodes in lysis buffer [10 $\mathrm{mM} \mathrm{NaH}_{2} \mathrm{PO}_{4}$ (pH 7.5), $100 \mathrm{~mm} \mathrm{NaCl}, 12 \mathrm{~mm}$ deoxycholate, $0.01 \%$ BSA, $1 \%$ Triton X-100, $0.1 \%$ SDS, $250 \mathrm{~mm}$ iodoacetamide] and then precipitating with polyclonal anti-actomyosin antibodies (R. Francis and R.H. Waterston, unpubl.) and Staph A (Kessler 1975). Samples were electrophoresed on 3.75\% SDS-polyacrylamide gels using the buffer system of Neville (1971). Proteins were visualized by silver staining (Wray et al. 1981). A Bio-Rad GS-300 scanning densitometer was used to 
estimate the MHC B levels. Individual lanes were compared to each other by normalizing the MHC B band intensity to that of the pharyngeal MHC C plus D, which are constant (Waterston et al. 1982). Several observations indicate that immunoprecipitation of the MHC was quantitative: (1) the relative proportions of wild-type MHC A, B, C, and D agreed well with published values (Garcea et al. 1978; Waterston et al. 1982); (2) the approximately twofold increase in MHC A level caused by sup-3 mutations (Waterston et al. 1982) was evident in all cases; and (3) repetitions of the experiments yielded reproducible results. The MHC B band of unc-54(r342) is barely detectable, even by eye. Based on its densitometry, we estimate that our limits of detection are $\sim 2 \%$ of wild-type MHC B levels.

\section{Northern blot analysis}

Total RNAs were extracted from mixed populations of worms by the method of Ross (1976) in the presence of vanadylribonucleosides. These RNAs were glyoxylated, separated on a $0.8 \%$ agarose gel, and transferred to a Nytran filter. Hybridizations were done according to Maniatis et al. (1982), with only minor modifications. The hybridization probes were radiolabeled by nick-translation (Rigby et al. 1977). We used hybridization probes SG23/1 and SG25/3 for unc-54 (MacLeod et al. 1981) and pCeA7 for act-1,2,3,4 controls (Landel et al. 1984).

\section{Acknowledgments}

We thank Ross Francis for the gift of anti-actomyosin antibodies. We are grateful to our colleagues R. A. Drysdale, T. Schedl, and E. Spangler for their helpful comments on the manuscript. We extend special thanks to Renate Bromberg for her help with electron microscopy. This work was supported by a National Institutes of Health Individual Research Grant (GM-30132) and by the University of Wisconsin Training Grant in Genetics.

\section{References}

Anderson, P. and S. Brenner. 1984. A selection for myosin heavy-chain mutants in the nematode C. elegans. Proc. Natl. Acad. Sci. 81: 4470-4474.

Bautch, V.L. and R.V. Storti. 1983. Identification of a cytoplasmic tropomyosin gene linked to two muscle tropomyosin genes in Drosophila. Proc. Natl. Acad. Sci. 80: $7123-7127$.

Bejsovec, A., D. Eide, and P. Anderson. 1984. Genetic techniques for analysis of nematode muscle. In Molecular biology of the cytoskeleton (ed. G.G. Borisy, D.W. Cleveland, and D.B. Murphyl, pp. 267-273. Cold Spring Harbor Laboratory, Cold Spring Harbor, New York.

Bennett, P., R. Craig, R. Starr, and G. Offer. 1986. The ultrastructural location of C-protein, X-protein, and H-protein in rabbit muscle. I. Muscle Res. and Cell Motil. 7: 550-567.

Brenner, S. 1974. The genetics of Caenorhabditis elegans. Genetics 77: 71-94.

Clark, S.H., A.J. Hilliker, and A. Chovnick. 1986. Genetic analysis of the right $\left(3^{\prime}\right)$ end of the rosy locus of Drosophila melanogaster. Genetical Res. 47: 109-116.

Craig, R., R. Padron, and J. Kendrick-Jones. 1987. Structural changes accompanying phosphorylation of tarantula muscle myosin filaments. J. Cell Biol. 105: 1319-1327.

Deak, I.I., P.R. Bellamy, M. Bienz, Y. Dubuis, E. Fenner, M. Gollin, A. Rahmi, T. Ramp, C.A. Reinhardt, and B. Cotton. 1982. Mutations affecting the indirect flight muscles of Drosophila melanogaster. I. Embryol. Exp. Morph. 69: 6181 .
Dibb, N.J., D.M. Brown, J. Karn, D.G. Moerman, S.L. Bolten, and R.H. Waterston. 1985. Sequence analysis of mutations that affect the synthesis, assembly, and enzymatic activity of unc-54 myosin heavy chain of C. elegans. J. Mol. Biol. 183: $543-551$.

Epstein, H.F., D.M. Miller, L.A. Gossett, and R.M. Hecht. 1982. Immunological studies of myosin isoforms in nematode embryos. In Muscle development, molecular and cellular control (ed. M.L. Pearson and H.F. Epstein). pp. 7-14. Cold Spring Harbor Laboratory, Cold Spring Harbor, New York.

Epstein, H.F., D.M. Miller, I. Ortiz, and G.C. Berliner. 1985. Myosin and paramyosin are organized about a newly identified core structure. J. Cell Biol. 100: 904-915.

Epstein, H.F., R.H. Waterston, and S. Brenner. 1974. A mutant affecting the heavy chain of myosin in Caenorhabditis elegans. J. Mol. Biol. 90: 291-300.

Francis, G.R. and R.H. Waterston. 1985. Muscle organization in C. elegans: Localization of proteins implicated in thin filament attachment and I-band organization. J. Cell Biol. 101: $1532-1549$.

Fyrberg, E.A., K.L. Kindle, N. Davidson, and A. Sodja. 1980. The actin genes of Drosophila: a dispersed multigene family. Cell 19: 365-378.

Garcea, R.L., F. Schachat, and H.F. Epstein. 1978. Coordinate synthesis of two myosins in wild-type and mutant nematode muscle during larval development. Cell 15: 421-428.

Goldberg, A.L. and A.C. St. John. 1976. Intracellular protein degradation in bacteria and membrane cells. Part 2. Annu. Rev. Biochem. 45: 747-803.

Greenwald, I.S. and H.R. Horvitz. 1980. unc-93(e1500): A behavior mutant of Caenorhabditis elegans that defines a gene with a wild-type null phenotype. Genetics 96: 147-164.

Hershko, A. and A. Ciechanover. 1982. Mechanisms of intracellular protein breakdown. Annu. Rev. Biochem. 51: 335364.

Hiromi, Y. and Y. Hotta. 1985. Actin gene mutations in Drosophila; heat shock activation in the indirect flight muscles. EMBO J. 4: 1681-1687.

Jarvick, J. and D. Botstein. 1973. A genetic method for determining the order of events in a biological pathway. Proc. Natl. Acad. Sci. 70: 2046-2050.

Karlik, C.C., M.D. Coutu, and E.A. Fyrberg. 1984. A nonsense mutation within the act $88 \mathrm{~F}$ actin gene disrupts myofibril formation in Drosophila indirect flight muscles. Cell 38: $711-719$.

Kemphues, K.J., R.A. Raff, T.C. Kaufman, and E.C. Raff. 1979. Mutation and structural gene for a b-tubulin specific to testis in Drosophila melanogaster. Proc. Natl. Acad. Sci. 76: 3991-3995.

Kendrick-Jones, J., P. Tooth, K.A. Taylor, and M. Scholey. 1982. Regulation of myosin filament assembly by light chain phosphorylation. Cold Spring Harbor Symp. Quant. Biol. 46: 929-938.

Kessler, S.W. 1975. Rapid isolation of antigens from cells with a staphylococcal protein A-antibody adsorbent: parameters of the interaction of antibody-antigen complexes with protein A. I. Immunology 115: 1617-1624.

Kiehart, D.P., D.A. Kaiser, and T.D. Pollard. 1984. Direct localization of monoclonal antibody-binding sites on Acanthamoeba myosin-II and inhibition of filament formation by antibodies that bind to specific sites on the myosin-II tail. $J$. Cell Biol. 99: 1015-1023.

Kuznicki, J., G.P. Cote, B. Bowers, and E.D. Korn. 1985. Filament formation and actin-activated ATPase activity are abolished by proteolytic removal of a small peptide from the tip of the tail of the heavy chain of Acanthamoeba myosin II. J. Biol. Chem. 260: 1967-1972. 
Landel, C.P., M. Krause, R.H. Waterston, and D. Hirsh. 1984. DNA rearrangements of the actin gene cluster in Caenorhabditis elegans accompany reversion of three muscle mutants. I. Mol. Biol. 180: 497-513.

Levitan, M. 1988. Textbook of human genetics, 3rd edition, pp. 475. Oxford University Press, New York.

Luck, D.J., B. Huang, and G. Piperno. 1982. Genetic and biochemical analysis of the eukaryotic flagellum. Symp. Soc. Exp. Biol. 35: 399-419.

Mackenzie, J.M. Jr., F. Schachat, and H.F. Epstein. 1978. Immunocytochemical localization of two myosins within the same muscle cells in Caenorhabditis elegans. Cell 15: 413419.

MacLeod, A.R., J. Karn, and S. Brenner. 1981. Molecular analysis of the unc-54 myosin heavy chain gene of Caenorhabditis elegans. Nature 291: 386-390.

MacLeod, A.R., R.H. Waterston, R.M. Fishpool, and S. Brenner. 1977. Identification of the structural gene for a myosin heavy-chain in C. elegans. J. Mol. Biol. 114: 133-140.

Maniatis, T., E.F. Fritsch, and J. Sambrook. 1982. Molecular cloning: a laboratory manual, pp. 545. Cold Spring Harbor Laboratory, Cold Spring Harbor, New York.

Maruyama, K., T. Yoshioka, H. Higuchi, K. Ohashi, S. Kiumra, and R. Natori. 1985. Connectin filaments link thick filaments and Z-lines in frog skeletal muscle as revealed by immunoelectron microscopy. J. Cell Biol. 101: 2167-2172.

McLachlan, A.D. and J. Karn. 1982. Periodic charge distributions in the myosin rod amino acid sequence match crossbridge spacings in muscle. Nature 299: 226-231.

- 1983. Periodic features in the amino acid sequence of nematode myosin rod. J. Mol. Biol. 164: 605-626.

Miller, D.M. and I. Maruyama. 1986. The sup-3 locus is closely linked to a myosin heavy chain gene in C. elegans. In Molecular biology of development, UCLA Symposia on Molecular and Cellular Biology, vol. 29 (ed. C. Emerson, D. Fischman, B. Nadal-Ginard, and M.A.Q. Siddiqui), pp. 629-638. Alan R. Liss, New York.

Miller, D.M., I. Ortiz, G.C. Berliner, and H.F. Epstein. 1983. Differential localization of two myosins within nematode thick filaments. Cell 34: 477-490.

Miller, D.M., F.E. Stockdale, and J. Karn. 1986. Immunological identification of the genes encoding the four myosin heavy chain isoforms of Caenorhabditis elegans. Proc. Natl. Acad. Sci. 83: 2305-2309.

Minty, A.J., S. Alonso, J.L. Guenet, and M. Buckingham. 1983. Number and organization of actin-related sequences in the mouse genome. I. Mol. Biol. 167: 77-101.

Moerman, D.G., S. Plurad, R.H. Waterston, and D.L. Baillie. 1982. Mutations in the unc-54 myosin heavy chain gene of C. elegans that alter contractility but not muscle structure. Cell 29: 773-781.

Mogami, K. and Y. Hotta. 1981. Isolation of Drosophila flightless mutants which affect myofibrillar proteins of indirect flight muscle. Mol. Gen. Genet. 183: 409-417.

Mogami, K., P.T. O'Donnell, S.I. Bernstein, T.R.F. Wright, and C.P. Emerson Jr. 1986. Mutations of the Drosophila myosin heavy chain gene: Effects on transcription, myosin accumulation, and muscle function. Proc. Natl. Acad. Sci. 83: 1393-1397.

Muller, H.J. 1932. Further studies on the nature and causes of gene mutations. In Proceedings of the sixth int. congress of genetics, vol. 1, Transactions and General Addresses, pp. 213-255. Brooklyn Botanic Garden, Brooklyn, New York.

Neville, D.M., Jr. 1971. Molecular weight determination of protein-dodecyl sulfate complexes by gel electrophoresis in a discontinuous buffer system. I. Biol. Chem. 246: 63286334.
Niederman, R. and L.K. Peters. 1982. Native bare zone assemblage nucleates myosin filament assembly. I. Mol. Biol. 161: $505-517$.

Pagh, K. and G. Gerisch. 1986. Monoclonal antibodies binding to the tail of Dictyostelium discoideum myosin: their ef fects on antiparallel and parallel assembly and actin-activated ATPase activity. J. Cell Biol. 103: 1527-1538.

Park, E.-C. and H.R. Horvitz. 1986. C. elegans unc-105 mutations affect muscle and are suppressed by other mutations that affect muscle. Genetics 113: 853-867.

Peltz, G., J.A. Spudich, and P. Parham. 1985. Monoclonal antibodies against seven sites on the head and tail of Dictyostelium myosin. J. Cell Biol. 100: 1016-1023.

Riddle, D.L. and S. Brenner. 1978. Indirect suppression in $C$. elegans. Genetics 89: 299-314.

Rigby, P.W.J., M. Diekmann, C. Rhodes, and P. Berg. 1977. Labeling deoxyribonucleic acid to high specific activity in vitro by nick translation with DNA polymerase I. $J$. Mol. Biol. 113: 237-251.

Romans, P. and R.A. Firtel. 1985. Organization of the actin multigene family of Dictyostelium discoideum and analysis of variability in the protein coding regions. I. Mol. Biol. 186: $321-335$.

Rosenbluth, J. 1965. Structural organization of obliquely striated muscle fibers in Ascaris lumbricoides. J. Cell Biol. 25: 495-515.

Ross, J. 1976. A precursor of globin messenger RNA. I. Mol. Biol. 106: 403-420.

Sanger, J.W., B. Mittal, and J.M. Sanger. 1984. Analysis of myofibrillar structure and assembly using fluorescently labeled contractile proteins. I. Cell Biol. 98: 825-833.

Schachat, F., R.L. Garcea, and H.F. Epstein. 1978. Myosins exist as homodimers of heavy chains: Demonstration with specific antibody purified by nematode mutant myosin affinity chromatography. Cell 15: 405-411.

Squire, J. 1981. The structural basis of muscular contraction, Plenum Press, New York.

Suzuki, D. 1970. Temperature-sensitive mutations in Drosophila melanogaster. Science 170: 695-706.

Suzuki, H., H. Onishi, K. Takahashi, and S. Watanabe. 1978. Structure and function of chicken gizzard myosin. $J$. Biochem. 84: 1529-1542.

Waterston, R.H., D. Hirsh, and T.R. Lane. 1984. Dominant mutations affecting muscle structure in Caenorhabditis elegans that map near the actin gene cluster. J. Mol. Biol. 180: $473-496$.

Waterston, R.H., D.G. Moerman, D. Baillie, and T.R. Lane. 1982. Mutations affecting myosin heavy chain accumulation and function in the nematode Caenorhabditis elegans. In Disorders of the motor unit (ed. D.L. Shotland), pp. 747-759. John Wiley, New York.

Waterston, R.H., J.N. Thomson, and S. Brenner. 1980. Mutants with altered muscle structure in Caenorhabditis elegans. Dev. Biol. 77: 271-302.

Wood, W.B. and J. King. 1979. Genetic control of complex bacteriophage assembly. In Comprehensive virology (ed. $\mathrm{H}$. Fraenkel-Conrat and R. Wagner), pp. 581-633. Plenum Press, New York.

Wray, W., T. Boulikas, V. Wray, and R. Hancock. 1981. Silver staining of proteins in polyacrylamide gels. Anal. Biochem. 118: 197-203.

Zengel, J.M. and H.F. Epstein. 1980. Identification of genetic elements associated with muscle structure in the nematode Caenorhabditis elegans. Cell Motility 1: 73-97. 


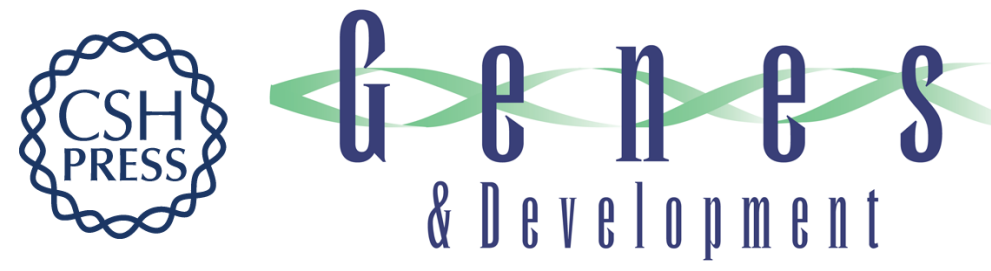

\section{Myosin heavy-chain mutations that disrupt Caenorhabditis elegans thick filament assembly.}

A Bejsovec and $P$ Anderson

Genes Dev. 1988, 2:

Access the most recent version at doi:10.1101/gad.2.10.1307

References This article cites 56 articles, 23 of which can be accessed free at:

http://genesdev.cshlp.org/content/2/10/1307.full.html\#ref-list-1

License

Email Alerting

Service

Receive free email alerts when new articles cite this article - sign up in the box at the top right corner of the article or click here.

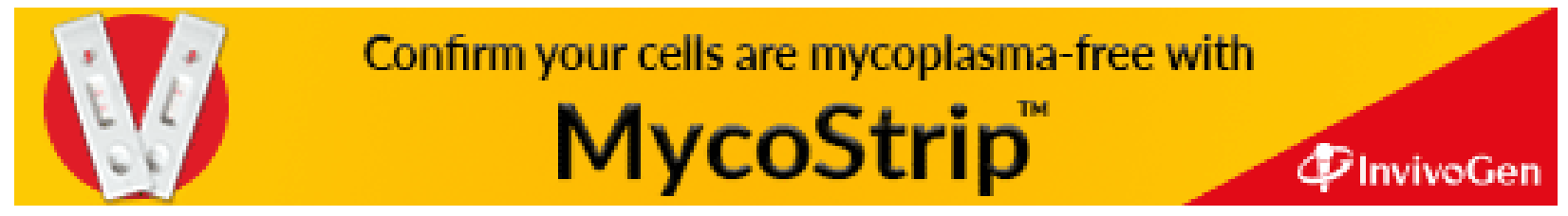

